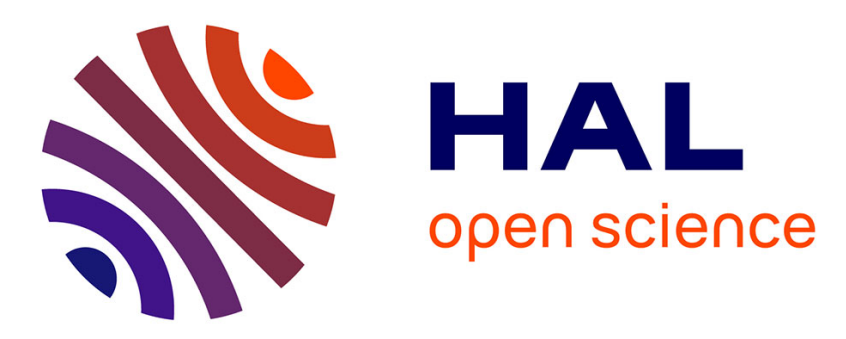

\title{
Electric dipole radiation at vlf in a uniform warm magneto-plasma
}

\author{
T.N.C. Wang, T.F. Bell
}

\section{To cite this version:}

T.N.C. Wang, T.F. Bell. Electric dipole radiation at vlf in a uniform warm magneto-plasma. Revue de Physique Appliquée, 1972, 7 (1), pp.11-20. 10.1051/rphysap:019720070101100 . jpa-00243589

\section{HAL Id: jpa-00243589 https://hal.science/jpa-00243589}

Submitted on 1 Jan 1972

HAL is a multi-disciplinary open access archive for the deposit and dissemination of scientific research documents, whether they are published or not. The documents may come from teaching and research institutions in France or abroad, or from public or private research centers.
L'archive ouverte pluridisciplinaire HAL, est destinée au dépôt et à la diffusion de documents scientifiques de niveau recherche, publiés ou non, émanant des établissements d'enseignement et de recherche français ou étrangers, des laboratoires publics ou privés. 


\title{
ELECTRIC DIPOLE RADIATION AT VLF IN A UNIFORM WARM MAGNETO-PLASMA
}

\author{
T. N. C. WANG \\ Radio Physics Laboratory, Stanford Research Institute, Menlo Park, Calif., USA \\ T. F. BELL \\ Radioscience Laboratory, Stanford University, Stanford, Calif., USA
}

(Reçu le 20 septembre 1971)

\begin{abstract}
Résumé. - Cet article traite des caractéristiques de rayonnement à très basse fréquence d'une antenne dipolaire mince dans un magnétoplasma tiède. Les solutions formelles sont obtenues pour la partie de la résistance de rayonnement due aux modes thermiques $\left(R^{\mathrm{t}}\right)$ et au mode sifflement $\left(R^{\mathrm{w}}\right)$. Dans ce calcul, les ions sont supposés au repos, et les effets de température non nulle sont introduits au moyen d'un terme de pression scalaire. Deux orientations particulières du dipôle sont prises en considération (orientations parallèle et perpendiculaire à la ligne de force du champ magnétique statique). Une analyse détaillée est effectuée pour les fréquences comprises entre la fréquence gyromagnétique électronique $\left(f_{\mathrm{He}}\right)$ et la fréquence de résonance hybride inférieure $\left(f_{\mathrm{LHR}}\right)$. Plusieurs graphes numériques de $R_{\|, \perp}^{\mathrm{t}}$ et $R_{\|, \perp}^{\mathrm{w}}$ sont présentés pour des valeurs typiques des paramètres du plasma magnétosphérique aux très basses fréquences. Pour des fréquences $(f)$ éloignées de $f_{\mathrm{LHR}}$, $R_{\|, \perp}^{\mathrm{t}}$ est généralement inférieure à $2 \times 10^{-1} R_{\|, \perp}^{\mathrm{w}}$ tandis que pour $f \rightarrow f_{\mathrm{LHR}}, R_{\|, \perp}^{\mathrm{t}} \simeq 10^{-1} R \perp$ et $R_{\|}^{\mathrm{t}} \simeq R_{\|}^{\mathrm{w}}$. Utilisant ces valeurs numériques de $R_{\|, \perp}^{\mathrm{t}}$ et $R_{\|, \perp}^{\mathrm{w}}$, le rendement du rayonnement dipolaire $\eta^{\mathrm{w}}$ pour le mode sifflement a été évalué pour un certain nombre de cas particuliers. Dans la plupart des cas, $\eta^{\mathrm{w}} \gtrsim 70 \%$, mais décroît à $\sim 38 \%$ pour $f \simeq f_{\text {LHR }}$.
\end{abstract}

Abstract. - The present paper considers the VLF radiation characteristics from a thin dipole antenna in a warm magnetoplasma. Formal solutions are derived for the portions of the dipole radiation resistance due to the thermal modes $\left(R^{t}\right)$ and the whistler mode $\left(R^{\mathrm{w}}\right)$. In this derivation the ions are assumed to be cold, and the effects of finite electron temperatures are included by means of a scalar pressure term. Two particular orientations of the dipole are considered (parallel and perpendicular to the static magnetic field line). A detailed analysis is made for frequencies in the range between the electron gyrofrequency $\left(f_{\mathrm{He}}\right)$ and the lower-hybrid-resonance frequency $\left(f_{\mathrm{LHR}}\right)$. Several numerical plots of $R_{\|, \perp}^{\mathrm{t}}$ and $R_{\|, \perp}^{\mathrm{w}}$ are presented for typical VLF magnetospheric plasma parameters. They show that for frequencies $(f)$ not close to $f_{\mathrm{LHR}}, R_{\|, \perp}^{t}$ is generally $<2 \times 10^{-1} R_{\|, \perp}^{\mathrm{w}}$ whereas as $f \rightarrow f_{\mathrm{LHR}}, R_{\perp, \perp}^{\mathrm{t}} \simeq 10^{-1} R_{\perp}^{\mathrm{w}}$ and $R_{\|}^{\mathrm{t}} \simeq R_{\|}^{\mathrm{W}}$. Using these numerical values of $R_{\|, \perp}^{\mathrm{t}}$ and $R_{\|, \perp}^{w}$, the dipole whistler mode radiation efficiences $\eta^{\text {w }}$ have been estimated for a number of specific cases. In most cases $\eta^{\mathrm{w}} \gtrsim 70 \%$, but falls as low as $\sim 38 \%$ at $f \simeq f_{\mathrm{LHR}}$.

I. Introduction. - A strong interest currently exists in the study of VLF antenna radiation characteristics in a magnetoplasma because of the application of this study to satellite-based wave-particle interaction experiments in the magnetosphere, to satellite communications systems and to ionospheric diagnostics. Most theoretical work on VLF antenna radiation in a magnetoplasma has so far been done under the assumption that the plasma is cold [1-21], and only a few workers have considered the effects of finite temperature in the antenna radiation characteristics [21-27]. The bulk of the knowledge on this subject can be found in a number of recent review articles such as [28 or 29].

The inclusion of a finite temperature in the magnetoplasma may vastly complicate the problem of antennamagnetoplasma coupling. A number of phenomena related to antenna properties can occur. In the $\mathrm{VLF} / \mathrm{ELF}$ range, there is a thermal conductance directly related to the thermal flux of plasma particles towards the antenna surface and in addition, there is a reactance associated with changes in the sheath surrounding the antenna elements [30]. These two factors contribute to make up the antenna sheath impedance [31].

A second effect of finite temperature is that waves may exist in a warm magnetoplasma that have no counterpart in the cold magnetoplasma. These thermal waves can take part in energy storage as well as energy propagation (also dissipation if the mode is subject to any kind of damping) from the antenna and thus these thermal modes will have an effect upon the antenna's impedance.

A third thermal effect at VLF is that near the wave 
normal range where the cold-plasma whistler mode phase velocity is comparable to the thermal velocities of the particles, e. g., in the vicinity of the cold plasma resonance cone, the propagation characteristics of the warm plasma whistler mode may be drastically altered from that of the cold plasma whistler mode. This «thermally modified" whistler mode (TMW) is electrostatic in nature and can propagate at wave normal angles greater than the cold plasma resonance wave angle.

With the appearance of the thermal modes and the thermally modified whistler mode in a warm magnetoplasma system, it is anticipated that a certain amount of power from the antenna will be radiated into these modes (if they are propagating). In view of their large refractive indices, the wave lengths of these modes may be expected to be of the order of the Debye length and thus it is possible that strong Landau or cyclotron damping may occur for these modes [32]. Collisional damping may also become important in regard to the propagation of these modes. A partial discussion of the effects of collisional damping has been given by [22] Due to the presence of these two damping mechanisms, it is most likely that the power radiated into the thermal modes and the thermally modified whistler mode cannot be extracted in the far zone. Thus these portions of dipole power may be considered as losses. In the planning of satellite VLF transmitter experiments a question then arises concerning the efficiency of the dipole in radiating whistler mode waves which can appear in the far field. To answer this question, it is necessary to estimate the amount of power radiated into thermal modes and/or the " thermally modified" whistler mode, or, equivalently, to calculate the dipole radiation resistance for these modes.

The purpose of the present paper is to calculate the whistler mode radiation efficiency of a dipole antenna in a warm, uniform magnetoplasma of infinite extent. To simplify the analysis, we omit the nonlinear problem of the antenna sheath and assume that the plasma surrounding the dipole antenna is entirely uniform and that no current flows from the plasma to the antenna. It is also assumed that the antenna voltage operation level is low enough so that a linear analysis will apply. Furthermore it is assumed that the macroscopic effects of thermal motion can be described by the adiabatic theory with scalar pressure terms [22], [24].

In calculating the dipole radiation resistance for the thermal modes and the thermally modified whistler mode our analysis includes the finite temperature only for the electrons. The finite temperatures of the ions are not considered because in general the ion thermal modes are heavily damped [32] and thus should contribute mainly to the reactive power flow.

Since it is expected that the thermal effects become most important for the case in which the cold-plasma whistler mode refractive index becomes quite large, we calculate the thermal mode radiation resistance only over the frequency range between the lower-hybrid- resonance frequency $\left(f_{\text {LHR }}\right)$ and the electron gyrofrequency $\left(f_{\mathrm{He}}\right)$ where the cold plasma whistler modes possesses a resonance cone. For the case of $f_{\mathrm{Hp}}<f<f_{\mathrm{LHR}}\left(f_{\mathrm{Hp}}=\right.$ proton gyrofrequency), the refractive index surface of the cold plasma whistler mode is closed and in general the phase velocity of the whistler mode will be much larger than the particle thermal velocities. Hence in this frequency range the characteristics of the cold-plasma whistler mode should not be drastically altered by the presence of a small temperature in the plasma.

In deriving the formal solution of the warm plasma dipole input impedance a full-wave analysis is used and two antenna orientations are considered, parallel and perpendicular to the static magnetic field. A general dispersion equation governing the modes of propagation is derived and a detailed analysis is made of the propagation characteristics of these modes. Numerical calculations of the thermal mode radiation resistance are made for the frequency ranges $f_{\mathrm{He}}>f \gg f_{\mathrm{LHR}}$ and $f \simeq f_{\mathrm{LHR}}$. From these numerical results the dipole whistler-mode radiation efficiencies are estimated for a number of cases applicable to the magnetosphere.

II. Fourier transformed electric field. - To derive an expression of the Fourier transformed electric field from a source embedded in a warm multicomponent magnetoplasma, we consider a case in which :1) the thermal motion of ions can be ignored ; 2) the macroscopic effects of the electron thermal motion are described by the transport theory with a scalar electron pressure. The basic equations governing the electromagnetic radiation from a time harmonic $\left(\mathrm{e}^{j \omega t}\right)$, smallamplitude (linear theory) source immersed in the uniform, multicomponent, warm-electron magnetoplasma are given by (in RMKS units) :

$$
\begin{aligned}
& \nabla \times \mathbf{E}=-j \omega \mu_{0} \mathbf{H} \\
& \nabla \times \mathbf{H}=j \omega \varepsilon \mathbf{E}-|e| N_{e} \mathbf{v}_{e}+\sum_{s} q_{s} N_{s} \mathbf{v}_{s}+\mathbf{J} \\
& j \omega \mathbf{v}_{e}=-\frac{|e|}{m_{e}}\left(\mathbf{E}+\mathbf{v}_{e} \times \mathbf{B}_{0}\right)-\frac{\nabla P_{e}}{N_{e} m_{e}} \\
& j \omega \mathbf{v}_{s}=\frac{q_{s}}{m_{s}}\left(\mathbf{E}+\mathbf{v}_{s} \times \mathbf{B}_{0}\right) \\
& u^{2} m_{e} N_{e} \nabla \cdot \mathbf{v}_{e}=-j \omega P_{e}
\end{aligned}
$$

where $\varepsilon, \mu_{0}, \omega, \mathbf{B}_{0}$ and $\mathbf{J}$ stand for free-space permittivity, free-space permeability, the angular signal frequency, the static magnetic field and the external current density, respectively, and where $\mathbf{v}_{s}, m_{s}, q_{s}$, and $N_{s}$ stand for the ordered velocity, the mass, thee charge and the unperturbed density for the " $S$ " species of particle, respectively, and where $P_{e}$ is the perturbed electron pressure and $u=\left(\gamma K T_{e} / m_{e}\right)^{\frac{1}{2}}$ is the thermal velocity of the electron gas.

Following an analysis similar to that given in 
chapter 3 of [21] or [17], a wave equation for the Fourier transformed electric field $\boldsymbol{\delta}(\mathbf{k})$ in terms of principal polarized wave coordinates can be deduced from (1)

$$
\begin{gathered}
\left(k^{2}-\beta^{2} \varepsilon_{v}\right) \delta_{v}-\alpha_{v} k_{v}(\mathbf{k} . \boldsymbol{E})= \\
=-\frac{j}{\omega \varepsilon}\left[\left(\alpha_{v}-1\right) k_{v}(\mathbf{k} . \mathfrak{J})+\beta^{2} J_{\mu} \delta_{v \mu}\right], \\
v=+1,-1,0
\end{gathered}
$$

where $: \beta=\omega / c$, the wave number in free space,

$$
\begin{gathered}
\alpha_{v}=1-\frac{\tilde{u}^{2}}{1-v Y}, \quad \tilde{u}=\frac{u}{c}, \quad Y=\frac{|e| B_{0}}{\omega m_{e}}, \\
\varepsilon_{v}=1-\sum_{s} \frac{X_{s}}{1+v Y_{s}},
\end{gathered}
$$

and $X_{s}$ and $Y_{s}$ (carrying sign) are standard notations for the normalized frequencies in the magneto-ionic theory, see, e. g., Ratcliffe [33].

In (2) $\mathfrak{J}$ stands for the Fourier transformation of $\mathbf{J}$ and $\mathfrak{J}_{\mu}(\mu=+1,-1,0)$ are the principal polarized components of $\mathfrak{J}$. Using (2) and the relation k. $\boldsymbol{E}=\sum_{\mu} k_{-\mu} \varepsilon_{\mu}, \mu=+1,-1,0$, the explicit form of k. $\boldsymbol{E}$ can be found

$$
\begin{gathered}
\mathbf{k} . \boldsymbol{E}=\frac{\pi(k)}{j \omega \varepsilon N(k)} \times \\
\times\left[\sum_{\mu} \frac{\beta^{2} k_{-\mu} J_{\mu}}{\left(k^{2}-\beta^{2} \varepsilon_{\mu}\right)}+(\mathbf{k} . \mathfrak{J}) \sum_{\mu} \frac{\left(\alpha_{\mu}-1\right) k_{\mu} k_{-\mu}}{\left(k^{2}-\beta^{2} \varepsilon_{\mu}\right)}\right]
\end{gathered}
$$

where :

$$
\begin{aligned}
\pi(k) & =\left(k^{2}-\beta^{2} \varepsilon_{+1}\right)\left(k^{2}-\beta^{2} \varepsilon_{-1}\right)\left(k^{2}-\beta^{2} \varepsilon_{0}\right) \\
N(k) & =C_{1} k^{6}+C_{2} \beta^{2} k^{4}+C_{3} \beta^{4} k^{2}+\beta^{6} C_{4} \\
C_{1} & =\tilde{u}^{2}\left(\frac{\sin ^{2} \theta}{1-Y^{2}}+\cos ^{2} \theta\right)
\end{aligned}
$$

( $\theta$ is the wave normal angle between $\mathbf{k}$ and $\mathbf{B}_{0}$ ),

$$
\begin{gathered}
C_{2}=-\tilde{u}^{2}\left[\frac{\left(\varepsilon_{0}+\varepsilon_{s}-\varepsilon_{d} Y\right)}{1-Y^{2}} \sin ^{2} \theta+2 \varepsilon_{s} \cos ^{2} \theta\right]-\alpha(\theta), \\
C_{3}=\tilde{u}^{2}\left[\frac{\varepsilon_{0}\left(\varepsilon_{s}-\varepsilon_{d} Y\right)}{1-Y^{2}} \sin ^{2} \theta+\varepsilon_{+1} \varepsilon_{-1} \cos ^{2} \theta\right]+ \\
+\varepsilon_{s}\left(2 \varepsilon_{0}+\left(a-\varepsilon_{0}\right) \sin ^{2} \theta\right) \\
C_{4}=-\varepsilon_{0} \varepsilon_{+1} \varepsilon_{-1} \quad \\
\alpha(\theta)=\varepsilon_{0} \cos ^{2} \theta+\varepsilon_{s} \sin ^{2} \theta \\
\varepsilon_{s}=\frac{1}{2}\left(\varepsilon_{+1}+\varepsilon_{-1}\right) \\
\varepsilon_{d}=\frac{1}{2}\left(\varepsilon_{+1}-\varepsilon_{-1}\right) \\
a=\frac{\varepsilon_{+1} \varepsilon_{-1}}{\varepsilon_{s}} .
\end{gathered}
$$

Inserting (3) into (2), the three principal components of the Fourier transformed electric field $\delta_{v}(\mathbf{k})(v=+1$, $-1,0)$ are given by

$$
\begin{aligned}
\varepsilon_{v}(\mathbf{k})=\frac{1}{j \omega \varepsilon} & {\left[\frac{\left(\alpha_{v}-1\right) k_{v}}{\left(k^{2}-\beta^{2} \varepsilon_{v}\right)}(\mathbf{k} . \mathfrak{J})+\frac{\beta^{2} \mathfrak{J}_{\mu} \delta_{v \mu}}{\left(k^{2}-\beta^{2} \varepsilon_{v}\right)}\right]+} \\
& +\frac{\alpha_{v} k_{v}}{j \omega \varepsilon\left(k^{2}-\beta^{2} \varepsilon_{v}\right)} \frac{\Pi(k)}{N(k)} \\
& \times\left[\sum_{\mu} \frac{\beta^{2} k_{-\mu} J_{\mu}}{\left(k^{2}-\beta^{2} \varepsilon_{\mu}\right)}+(\mathbf{k} . \mathfrak{J}) \sum_{\mu} \frac{\left(\alpha_{\mu}-1\right) k_{-\mu} k_{\mu}}{\left(k^{2}-\beta^{2} \varepsilon_{\mu}\right)}\right] .
\end{aligned}
$$

In case of $T_{\mathrm{e}}$ (electron temperature) $\rightarrow 0$, then $u \rightarrow 0$ and $\alpha_{v} \rightarrow 1$, and (4) reduces identically to eq. (12) of [17] for the case of a cold magnetoplasma.

III. Formal solution of input impedance. - The mean complex power radiated from a current source can be expressed [17]

$$
\begin{aligned}
P=-\frac{1}{16 \pi^{3}} \int \sum_{v}\left[J_{v}(-\mathbf{k})\right]^{*} \varepsilon_{v}(\mathbf{k}) \mathrm{d} \mathbf{k}, & \\
v & =+1,-1,0 .
\end{aligned}
$$

In this section, we make use of (4) and (5) to dervie a formal expression for the input impedance of a short dipole oriented either parallel or perpendicular to the static magnetic field. The dipole current is assumed to be a skin-triangular distribution.

A. Parallel orientation. - We center the dipole antenna of length $2 \mathrm{~h}$ and radius $r_{0}$ in a dextral Cartesian coordinate parallel to $\mathbf{B}_{0}$. The geometry of the problem is indicated in figure $1 a$. The dipole current (skin-triangular distribution) can be written in terms of principal polarized components ;

$$
\begin{aligned}
J_{0} & =I_{0}\left(1-\frac{|z|}{h}\right) \frac{\delta\left(\rho-r_{0}\right)}{2 \pi \rho}, \quad|z| \leqslant h \\
& =0, \quad|z| \geqslant h \\
J_{+1} & =J_{-1}=0
\end{aligned}
$$

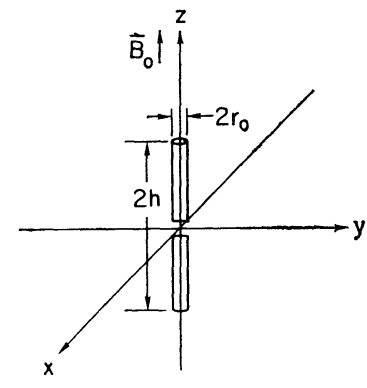

(a)

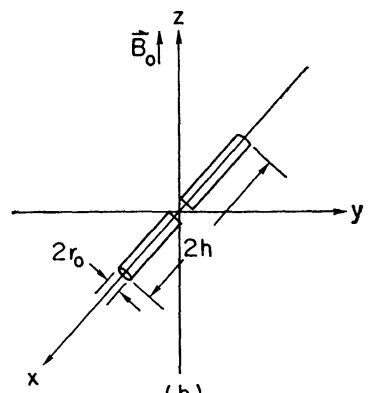

(b)
FIG. 1. - Geometrical configuration of the dipole antenna for the case of : $a$ ) parallel orientation and $b$ ) perpendicular orientation. 
where $\rho^{2}=x^{2}+y^{2}$ and where $I_{0}$ is the total current at the dipole driving terminals.

The Fourier transform of (6) is

$$
\begin{aligned}
\mathfrak{J}_{0}(\mathbf{k}) & =\frac{4 I_{0}}{h} \frac{\sin ^{2}\left(h k_{z} / 2\right)}{k_{z}^{2}} J_{0}\left(r_{0} k_{\perp}\right) \\
J_{+1}(\mathbf{k}) & =J_{-1}(\mathbf{k})=0
\end{aligned}
$$

where $k_{\perp}^{2}=k_{y}^{2}+k_{x}^{2}$.

Using (4), (5), (7) and the relation $Z=2 P / I_{0}^{2}$, the input impedance $Z_{\|}$for the parallel oriented dipole can be formally written

$Z_{\|}=\frac{j Z_{0}(h \beta)^{2}}{8 \pi^{3}} \int G_{\|}(n, \theta)\left[\frac{\sin \left(\lambda n_{z}\right)}{\lambda n_{z}}\right]^{4} J_{0}^{2}\left(\beta r_{0} n_{\perp}\right) \mathrm{d} \mathbf{n}$

where $\mathbf{n}=\mathbf{k} / \beta, \quad n_{\perp}^{2}=n_{x}^{2}+n_{y}^{2}, \quad Z_{0}=120 \pi$ ohms, $\lambda=h \beta / 2$,

$$
\begin{aligned}
& G_{\|}(n, \theta)= \\
& =\frac{1+\left(\alpha_{0}-1\right) n_{z}^{2}}{\left(n^{2}-\varepsilon_{0}\right)}\left[1+\frac{\alpha_{0} n_{z}^{2}\left(n^{2}-\varepsilon_{+1}\right)\left(n^{2}-\varepsilon_{-1}\right)}{N(n)}\right]+ \\
& +\frac{\alpha_{0} n_{z}^{2}}{N(n)}-n_{\perp}^{2}\left[\left(\alpha_{s}-1\right) n^{2}-\frac{1}{2}\left(\alpha_{+1} \varepsilon_{-1}+\alpha_{-1} \varepsilon_{+1}\right)+\varepsilon_{s}\right],
\end{aligned}
$$

$\alpha_{s}=\frac{1}{2}\left(\alpha_{+1}+\alpha_{-1}\right)$ and where $N(n)$ is obtained from $N(k)$ by substituting the identity $k=\beta n$, and multiplying the result by $\beta^{-6}$.

The input reactance and radiation resistance (for the lossless case considered here) are given respectively by the imaginary part and the real part of (8). A convenient method to extract the real and imaginary part of $Z_{\|}$is to perform a proper contour integration with respect to the variable $n$. It is clear from (8) that the residue contributions to the integral are due to the poles of $G_{\|}(n, \theta)$, namely $n^{2}=\varepsilon_{0}$, and the zeroes of $N(n)$

It is not difficult to show that the contribution in the contour integration due to the pole $\varepsilon_{0}$ vanishes identically [21]. The essential contribution to $Z_{\|}$therefore comes from those poles which are the zeroes of $N(n)$. The equation $N(n)=0$ is actually the dispersion relation of the warm magnetoplasma system considered. This dispersion relation is identical to that derived by [34] for a similar plasma model. The detailed analysis of this dispersion equation is given in the next section.

B. Perpendicular orientation. - For the case of dipole antenna oriented perpendicular to $\mathbf{B}_{0}$, we set the dipole along the $x$-axis. The geometrical configuration is indicated in figure $1 b$.

In this case the Fourier transformed dipole current in terms of principal polarized components takes the form ;

$$
\begin{aligned}
J_{+1}(\mathbf{k}) & =\frac{4 I_{0}}{\sqrt{2} h} \frac{\sin ^{2}\left(h k_{x} / 2\right)}{k_{x}^{2}} J_{0}\left(r_{0} k_{\perp}^{\prime}\right) \\
J_{0}(\mathbf{k}) & =0
\end{aligned}
$$

where $k_{\perp}^{\prime}=\sqrt{k^{2}-k_{x}^{2}}$.

Using (4), (5), (9) and the relation $Z_{\perp}=2 P_{\perp} / I_{0}^{2}$, the formal solution for the input impedance $Z_{\perp}$ can be expressed by

$$
Z_{\perp}=\frac{j Z_{0}(h \beta)^{2}}{16 \pi^{3}} \int G_{\perp}(n, \theta)\left[\frac{\sin \left(\lambda n_{x}\right)}{\lambda n_{x}}\right]^{4} J_{0}^{2}\left(\beta r_{0} n_{\perp}^{\prime}\right) \mathrm{d} \mathbf{n}
$$

where :

$$
\begin{aligned}
& G_{\perp}(n, \theta)= A_{+1}+A_{-1}+\frac{\Pi(n)}{N(n)}\left[\frac{\alpha_{+1} n_{+1}}{n^{2}-\varepsilon_{+1}}+\frac{\alpha_{-1} n_{-1}}{n^{2}-\varepsilon_{-1}}\right] \times \\
& \times {\left[n_{-1} A_{+1}+n_{+1} A_{-1}+\sqrt{2} n_{x} n_{z}^{2} \frac{\alpha_{0}-1}{n^{2}-\varepsilon_{0}}\right], } \\
& A_{ \pm 1}=\frac{1+\sqrt{2}\left(\alpha_{ \pm 1}-1\right) n_{x}}{n^{2}-\varepsilon_{ \pm 1}}, \\
& \Pi(n)=\beta^{-6} \Pi(k), n_{\perp}^{\prime}=\sqrt{n^{2}-n_{x}^{2}},
\end{aligned}
$$

and

$$
n_{ \pm 1}=\frac{1}{\sqrt{2}}\left(n_{x} \pm j n_{y}\right)
$$

The other notations in (10) have been defined in the previous section.

In (10) it can be shown that the contributions to $Z_{\perp}$ due to the poles $\varepsilon_{ \pm 1}$ vanish and thus the input impedance $Z_{\perp}$ arises only from the zeros of $N(n)$.

As $T_{\mathrm{e}} \rightarrow 0, \tilde{u} \rightarrow 0, \alpha_{v} \rightarrow 1$, it can be shown that (8) and (10) reduce to the correct cold magnetoplasma results [17 and 21]. It should be noted that the formal solutions of (8) and (10) are general ones which are valid for arbitrary values of driving frequency, plasma composition, particle density and static magnetic field.

Since our main concern in this paper is to calculate the power radiated from the dipole into the various characteristic modes in the frequency range between $f_{\mathrm{He}}$ and $f_{\mathrm{LHR}}$ ( $f_{\mathrm{He}}, f_{\mathrm{LHR}}$ stand for the electron gyrofrequency and lower-hybrid-resonance frequency, respectively) we shall restrict the analysis in the subsequent sections to the frequencies $f_{\mathrm{He}}>f \geqslant f_{\mathrm{LHR}}$.

IV. Analysis of the dispersion equation. - From (4), it can be shown that when one sets $J_{v}$ equal to zero, a non-trivial solution for $\varepsilon_{v}$ exists provided the condition $N(n)=0$ holds. Thus $N(n)=0$ is the dispersion equation which determines the propagating characteristic modes in the warm magnetoplasma system 
considered. The explicit form of the dispersion equation can be written ;

$$
C_{1} x^{3}+C_{2} x^{2}+C_{3} x+C_{4}=0
$$

where $x=n^{2}$.

Eq. (11) is equivalent to eq. (5.16) of [34]. Since (11) is a bicubic equation for the refractive index $n$, there exist three roots for $n^{2}$. In this section, we shall determine the positive roots of (11), corresponding to propagating modes, for the frequency range $f_{\mathrm{He}}>f \geqslant f_{\mathrm{LHR}}$. The plasma is assumed to have a relatively high density such that $f_{0}>f_{\mathrm{He}}\left(f_{0}\right.$ is the plasma frequency).

A. $f_{\mathrm{He}}>f>f_{q}\left(f_{0}>f_{\mathrm{He}}\right)$. - For the frequency range $f_{\mathrm{He}}>f>f_{q}\left(f_{q}\right.$ is defined below), an analysis of the roots of (11) (by the use of Descartes' Rule of signs and various related methods described in [35] yields the following results) :

1. There exists a unique real positive root of (11) for the angular range; $0 \leqslant \theta \leqslant \theta_{1}$, where $\theta_{1}=\arctan \sqrt{Y^{2}-1}$. Let $x_{\mathrm{r}}$ stand for this root. At $\theta=0, x_{\mathrm{r}}=\varepsilon_{+1}$ whereas at $\theta=\theta_{1}, x_{\mathrm{r}} \rightarrow \infty$. Note that when $\theta=0, x_{\mathrm{r}}$ is identical to the square of the cold plasma whistler mode refractive index.

2. There exist no real positive roots for the angular range $\theta_{1}<\theta<\pi / 2$. From this result together with the fact; $n_{\mathrm{r}}^{2} \rightarrow \infty$ as $\theta \rightarrow \theta_{1}$, it is clear that the angle $\theta_{1}$ serves as a new resonance cone angle for the unique propagating mode of the warm magnetoplasma system. (In the cold magnetoplasma, the whistler mode resonance cone is given by $\theta_{\mathrm{r}}=\arctan \sqrt{-\varepsilon_{0} / \varepsilon_{s}}$, see [17]. It can be shown that $\theta_{\mathrm{r}} \ll \theta_{1}$.)

3. As the wave normal angle $\theta$ increases, the effects of finite electron temperature become more important and $x_{\mathrm{r}}^{1 / 2}$ will gradually deviate from the cold plasma whistler mode refractive index. Eventually, as $\theta \rightarrow \theta_{1}$ thermal effects will dominate and $x_{\mathrm{r}}$ will differ markedly from the cold plasma results.

In addition to the angle $\theta_{1}$, there exists another angle which plays an important role in determining the characteristics of the roots and sets the lower bound of the frequency range considered in this section. This angle is given by the unique root of the relation $C_{2}(\theta)=0$ :

$$
\theta_{2}=\arctan \left[\frac{-\varepsilon_{0}-2 \varepsilon_{s} \tilde{u}^{2}}{\varepsilon_{s}+\frac{\tilde{u}^{2}\left(\varepsilon_{0}+\varepsilon_{s}-\varepsilon_{d} Y\right)}{1-Y^{2}}}\right]^{1 / 2}
$$

Note that as $\tilde{u} \rightarrow 0, \theta_{2} \rightarrow \theta_{\mathrm{r}}$, the resonance cone angle for cold plasma whistler mode.

It can be shown that for frequencies higher than $f_{q}$, $\theta_{2}$ is less than $\theta_{1}$. As frequency decreases (toward the lower-hybrid-resonance frequency) $\theta_{2}$ increases faster than $\theta_{1}$ and eventually exceeds $\theta_{1}$. For the case of $\theta_{2}>\theta_{1}$, the characteristics of the roots of (11) differ from the descriptions given in this section. The condition $\theta_{1}=\theta_{2}$ occurs at a frequency $f_{q}$, which serves as the lower bound of the frequency range in which the propagation characteristics described above are valid. Under the conditions ; $f_{0} / f_{\mathrm{He}}>\sqrt{2}$ and $\tilde{u}^{2} \ll 1, f_{q}$ for a proton-electron plasma is given approximately by

$$
f_{q}=\left[\frac{m_{\mathrm{p}}}{m_{\mathrm{e}}}\left(f_{\mathrm{He}}^{2} / f_{0}^{2}+2 \tilde{u}^{2}\right)\right]^{-1 / 2} f_{\mathrm{He}}
$$

where $m_{\mathrm{p}}$ is the proton mass.

From the above results, it is clear that within the frequency range $f_{q}<f<f_{\mathrm{He}}$, only one propagating mode exists in the wave normal range $0<\theta<\theta_{1}$. The refractive index $n$ of this mode is identical to that of the cold plasma whistler mode at $\theta=0$. As the wave normal angle $\theta$ increases, $n$ gradually deviates from $n_{-}(\theta)$ (cold plasma whistler mode refractive index). When the wave normal angle $\theta$ reaches a certain value close to $\theta_{\mathrm{r}}$, say $\theta_{\mathrm{cr}}\left(\theta_{\mathrm{cr}}<\theta_{1}\right), n$ starts to differ markedly from $n_{-}(\theta)$. To make a simple description of the propagation structure, it is convenient to divide the angular range of propagation $0<\theta<\theta_{1}$ into two separate regions; $0<\theta<\theta_{\mathrm{cr}}$ and $\theta_{\mathrm{cr}}<\theta<\theta_{1}$. The range $0<\theta<\theta_{\text {cr }}$ in which the whistler mode is relatively unaffected by temperature we define as the region for normal whistler mode propagation whereas the range $\theta_{\text {cr }}<\theta<\theta_{1}$, in which $n$ differs markedly from $n_{-}(\theta)$, we define as the region in which a «thermally modified " whistler mode (TMW) propagates. Although we have defined here two separate regions for propagation, it should be emphasized that the transition from the normal whistler mode to the TMW mode is a continuous one and thus the choice of any given value for $\theta_{\mathrm{cr}}$ is somewhat arbitrary. For the purposes of the present paper we choose $\theta_{\mathrm{cr}} \simeq \theta_{<}$. The reason for this choice is that in general for angles greater than $\theta_{2}$ the conditions for heavy Landau or cyclotron damping are satisfied (i. e., $\tilde{u} n \cos \theta \simeq 1-s Y_{\mathrm{e}} ; s=0, \pm 1$ ), while for $\theta<\theta_{2}$ these conditions, in general, are not satisfied ; thus the lightly damped mode propagating in the angular range $\theta<\theta_{2}$ can contribute to the far-field power flow while the heavily damped mode in the angular range $\theta>\theta_{2}$ should not contribute significantly to the far-field power flow. The choice of $\theta_{\text {cr }} \simeq \theta_{2}$ becomes questionable whenever $f \simeq f_{q}$ since in this case $\theta_{1} \simeq \theta_{2}$ and the angular range of propagation of the TMW mode would be forced to zero. However in the present paper we do not consider frequencies in the range $f \approx f_{q}$ and this problem is avoided. A qualitative diagram for the mode propagation described here is indicated in figure $2 a$. For the sake of brevity, the diagrams (similar to that given in [34]) of the phase velocities for the various modes described in figure 2 are omitted.

(The results presented above are quite different from those of [22]. Using a similar warm magnetoplasma model, Kieburtz obtained a perturbational solution 
for the dispersion equation and reached the conclusion that over a range of wave normal angles near $\theta_{1}$, there existed two distinct propagating modes. Kieburtz identified one of these modes as the whistler mode and the other mode, as the quasi-acoustic mode. Since, for the case of $m_{\mathrm{i}} \rightarrow \infty,(11)$ can be shown identical to the dispersion equation described by Kieburtz (eq. (10) of [22]), the results of the present work indicate that Kieburtz's approximate perturbation solutions are not valid near $\theta_{1}$.)

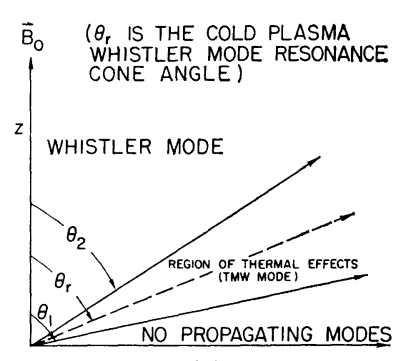

(a)

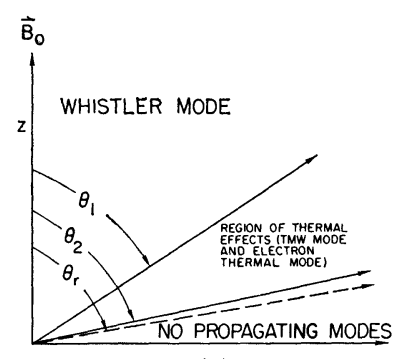

(b)
FIG. 2. - Qualitative diagram showing the angular regions in which the whistler mode and thermal modes propagate. Two frequency ranges are considered : a) $f_{\mathrm{He}}>f>f_{q}$ and b) $f_{q}>f \geqslant f_{\mathrm{LHR}}$.

B. $f_{q}>f \geqslant f_{\text {LHR }}$ - In the frequency range $f_{q}>f \geqslant f_{\mathrm{LHR}}, \theta_{2}>\theta_{1}$ and the propagation characteristics of the system can be summarized as follows :

1. For wave normal angles $0<\theta<\theta_{1}$ there exists a unique real positive root $x_{\mathrm{r}}(\theta)$. Numerical calculations show that $x_{\mathrm{r}}^{1 / 2}(\theta) / n_{-}(\theta) \simeq 1+0\left(10^{-2}\right)$ ( $T_{\mathrm{e}} \simeq 10^{4}{ }^{\mathrm{o}} \mathrm{K}$ is assumed) over the range $0<\theta<\theta_{1}$, as long as $f \approx f_{q}$. Thus for this wave normal range the original cold plasma whistler mode is essentially unaffected by the finite temperature.

2. For wave normal angles $\theta_{1}<\theta<\theta_{2}$, there are at most two real positive roots for (11). To determine the approximate value of the positive roots in the system we first extract the unique negative root which to a good approximation is given by

$$
x_{g} \simeq-\frac{C_{4}}{C_{3}} .
$$

After extracting the factor $n^{2}+C_{4} / C_{3}$ from (11) by long division and discarding the small remainder, a quadratic equation is obtained which has the two roots :

$n_{1,2}^{2}(\theta)=\frac{-B \pm \Delta}{2 C_{1}}\left(«+»\right.$ for $n_{1}^{2}$ and $«-»$ for $\left.n_{2}^{2}\right)$

where $B=\left(C_{2} C_{3}-C_{1} C_{4}\right) / C_{3}$ and

$\Delta=\left\{B^{2}-4 C_{1}\left[C_{3}-\left(\frac{C_{2} C_{4}}{C_{3}}-\frac{C_{1} C_{4}^{2}}{C_{3}^{2}}\right)\right]\right\}^{1 / 2}$.

It is possible to show that the relation $\Delta(\theta)=0$ has a unique solution $\theta_{c}$, in the angular range $\theta_{1}<\theta<\theta_{2}$ and that for $\theta_{1} \leqslant \theta \leqslant \theta_{\mathrm{c}}$ there are two real positive roots of (11), while for $\theta_{c}<\theta \leqslant \theta_{2}$ there are two complex conjugate roots. An examination of the two roots in (14) shows that $n_{1}$ can be identified as the TMW mode whereas $n_{2}$ is an electron thermal mode.

3. For the wave normal angles $\theta_{2}<\theta<\pi / 2$ the system has no positive real root and thus there are no propagating modes in this region.

A qualitative diagram for the mode propagation for this case is indicated in figure $2 b$.

V. Dipole radiation resistance due to the thermal modes (TMW mode and the electron thermal mode). In order to determine the whistler-mode radiation efficiency of the dipole antenna, it is necessary to estimate the amount of power radiated into the thermal modes, or, equivalently, to calculate the dipole radiation resistance for these modes. In view of the results obtained by analyzing the dispersion equation in the previous section, it is convenient to consider separately the dipole radiation resistance due to the thermal modes, $R^{t}$, over the frequency ranges $f_{q}>f \geqslant f_{\text {LHR }}$ and $f_{\mathrm{He}} \geqslant f>f_{q}$.

A. $R^{\mathrm{t}}$ IN THE FREQUENCY RANGE $f_{q}>f \geqslant f_{\mathrm{LHR}}$. In the frequency range $f \geqslant f \geqslant f_{\mathrm{LHR}}$, as discussed in the previous section, both a TMW and an electron thermal mode can propagate over the wave normal angles $\theta_{1} \leqslant \theta \leqslant \theta_{c}$.

Using (13) and (14) as the three roots of $N(n)$ and performing a contour integration in $n$, following a procedure similar to that discussed in earlier papers [17], [19], [20], the leading terms of the dipole radiation resistance due to the TMW and the electron thermal mode can be extracted from (8) and (10) for the two particular dipole orientations considered.

$$
\begin{aligned}
R_{\|}^{\mathrm{t}} \simeq \frac{4 Z_{0}}{\pi(h \beta)^{2}} \sum_{t=1}^{2} \int_{\theta_{1}}^{\theta_{\mathrm{c}}} \frac{\left(1-C_{1} n_{t}^{2}\right)}{n_{t} \Delta} \frac{\sin ^{4}\left(\frac{h \beta}{2} n_{t} \cos \theta\right)}{\cos ^{2} \theta} \times \\
\quad \times J_{0}^{2}\left(\beta r_{0} n_{t} \sin \theta\right) \sin \theta \mathrm{d} \theta \\
R_{\perp}^{\mathrm{t}=} \\
\quad \frac{4 Z_{0}}{\pi^{2}(h \beta)^{2}} \sum_{t=1}^{2} \int_{0}^{\pi / 2} \mathrm{~d} \psi \int_{\theta_{1}}^{\theta_{\mathrm{c}}} \frac{\left(1-C_{1} n_{t}^{2}\right)}{n_{t} \Delta} \times \\
\quad \times \frac{\sin ^{4}\left(\frac{\beta h}{2} n_{t} \sin \theta \cos \psi\right)}{\sin ^{2} \theta \cos ^{2} \psi} \frac{16}{} \\
\quad \times J_{0}^{2}\left(\beta r_{0} n_{t} \sqrt{\left.1-\sin ^{2} \theta \cos ^{2} \psi\right) \sin \theta \mathrm{d} \theta}\right.
\end{aligned}
$$

where $\Delta$ is defined in (15) and $n_{1,2}^{2}$ are defined in (14). In (16) a trivial integration with respect to the variable $\Psi$ has been performed.

The analytical integrations of $R_{\|}^{\mathrm{t}}$ and $R_{\perp}^{\mathrm{t}}$ are difficult. In order to make a quantitative comparison between $R_{\|, \perp}^{\mathrm{t}}$ and the whistler mode radiation resistance (to be 
discussed in the next section), (16) and (17) have been integrated numerically and the results are given in a later section.

B. $R^{\mathrm{t}}$ IN THE FREQUENCY RANGE $f_{\mathrm{He}}>f>f_{q}$. In the frequency range $f_{\mathrm{He}}>f>f_{q}$, as shown in section on analysis of the dispersion equation, the TMW mode propagates over the wave normal angles $\theta_{2} \leqslant \theta \leqslant \theta_{1}$. The refractive index of this mode is given approximately by the expression $n_{1}$ defined in (14). The total dipole radiation resistance due to this mode can be estimated following an analysis similar to that given in the previous section.

The leading term of the form results of $R_{\|}^{\mathrm{t}}$ and $R_{\perp}^{\mathrm{t}}$ can be shown to consist of the single integrals denoted in (16) and (17) as the terms $t=1$, given that the limits of integration are changed to $\left(\theta_{2}, \theta_{1}\right)$ instead of $\left(\theta_{1}, \theta_{\mathrm{c}}\right)$. The explicit written forms of $R_{\|}^{\mathrm{t}}$ and $R_{\perp}^{\mathrm{t}}$ are omitted for the sake of brevity. Numerical calculations of $R_{\|}^{\mathrm{t}}$ and $R_{\perp}^{\mathrm{t}}$ are given in a later section.

C. $R^{\mathrm{w}}$ IN THE FREQUENCY RANGE $f_{\mathrm{He}}>f>f_{q}$. To estimate the dipole radiation resistance $R$ due to the whistler mode for the warm magnetoplasma system considered, we note that in the frequency range $f_{\mathrm{He}}>f>f_{q}$ and within the wave normal range $0<\theta<\theta_{2}$, the refractive index for the unique propagating mode from $N(n)=0$ is essentially given by $n_{-}(\theta)$ (the refractive index for the cold plasma whistler mode). The effect of finite electron temperature can be shown to be unimportant in determining the roots of the dispersion equation. Therefore, to a good approximation we can let $\vec{u} \rightarrow 0$ in (11) and the three roots of (11) become $n_{\mathrm{et}}^{2} \rightarrow \infty$, and $n_{ \pm}^{2}(\theta)$, where $n_{ \pm}^{2}(\theta)$ (defined below) are identical to the two characteristic roots in the cold magnetoplasma. Using these three approximate roots of $N(n)$ and performing an analysis similar to that made in the previous section, the dipole radiation resistance $R_{\|, \perp}^{\mathrm{w}}$ due to the whistler mode can be extracted from (8) and (10). In the limit $\tilde{u} \ll 1$, the formal solution of $R_{\|, \perp}$ is given by :

$$
\begin{aligned}
R_{\|}^{\mathrm{w}}=\frac{(h \beta)^{2} Z_{0}}{4 \pi} \int_{0}^{\theta_{2}} \frac{n_{\|}^{2} n_{\perp}\left(n^{2}-\varepsilon_{+1}\right)\left(n^{2}-\varepsilon_{-1}\right)}{G(\theta)\left(n^{2}-\varepsilon_{0}\right)} \times \\
\\
\times\left[\frac{\sin \lambda n_{\|}}{\lambda n_{\|}}\right]^{4} J_{0}^{2}\left(\beta r_{0} n_{\perp}\right) \mathrm{d} \theta
\end{aligned}
$$

where $n_{\|}=n \cos \theta, \quad n_{\perp}=n \sin \theta, \quad n=n_{-}(\theta), \quad$ and where the other notations have been defined previously.

$$
\begin{aligned}
R_{\perp}^{\mathrm{w}}= & \frac{(h \beta)^{2} Z_{0}}{2 \pi^{2}} \int_{0}^{\pi / 2} \mathrm{~d} \psi \int_{0}^{\theta_{2}} \frac{n^{3}\left(n^{2}-\varepsilon_{0}\right) \sin ^{3} \theta}{G(\theta)} \times \\
& \times\left[\cos ^{2} \psi+\frac{\varepsilon_{\mathrm{d}}^{2}}{\left(n^{2}-\varepsilon_{+1}\right)\left(n^{2}-\varepsilon_{-1}\right)}\right] \\
& \times F(\psi, \theta) \mathrm{d} \theta,
\end{aligned}
$$

where

$$
\begin{aligned}
F(\psi, \theta) & =\left(\frac{\sin \lambda n_{x}}{\lambda n_{x}}\right)^{4} J_{0}^{2}\left(\beta r_{0} \sqrt{n^{2}-n_{x}^{2}}\right) \\
G(\theta) & =\sqrt{\left(\varepsilon_{0} \varepsilon_{s}-\varepsilon_{+1} \varepsilon_{-1}\right)^{2} \sin ^{4} \theta+4 \varepsilon_{0}^{2} \varepsilon_{d}^{2} \cos ^{2} \theta} \\
n_{x} & =n \sin \theta \cos \psi, \quad n=n_{-}(\theta) \\
n_{ \pm}^{2} & =\frac{1}{2}\left[\left(\varepsilon_{+1} \varepsilon_{-1}-\varepsilon_{0} \varepsilon_{s}\right) \sin ^{2} \theta+\right. \\
& \left.+2 \varepsilon_{0} \varepsilon_{s} \pm G(\theta)\right] \alpha^{-1}(\theta) .
\end{aligned}
$$

The integrals of (18) and (19) are almost identical to those appropriate to the case of a cold magnetoplasma (see eq. (23) and (B 1) of [17]). The only difference lies in the upper limit of $\theta$-integration. For the case of dipole embedded in a cold magnetoplasma, the upper limit of $\theta$-integration is the cold plasma whistler-mode resonance cone angle $\theta_{\mathrm{r}}$, whereas for the present case of warm magnetoplasma, the upper limit is given by $\theta_{2}$. The significance as well as the method for determining $\theta_{2}$ has been discussed in the section on analysis of the dispersion equation.

D. $R^{\mathrm{w}}$ IN THE FREQUENCY RANGE $f_{q}>f \geqslant f_{\text {LHR }}$ In the frequency range $f_{q}>f \geqslant f_{\mathrm{LHR}}$, it is clear from the section on analysis of the dispersion equation that in the wave normal range $0<\theta<\theta_{1}$, the cold plasma whistler mode is practically unaffected by the finite electron temperature. It can be shown that the formal solutions $R$, for this frequency range are again given by [17 and 19] except that the upper limit in $\theta$-integration is replaced by $\theta_{1}$, where $\theta_{1}=\arctan \sqrt{Y^{2}-1}$. In the case of a dipole in a cold magnetoplasma the upper limit is the angle $\theta_{\mathrm{r}}$, which is identical to $\pi / 2$ as $f \rightarrow f_{\mathrm{LHR}}\left(\varepsilon_{s} \rightarrow 0\right)$. The relevant integrals of $R_{\|, \perp}^{\mathrm{w}}$ for the cold plasma can be shown to have a nonintegrable singularity at $f=f_{\mathrm{LHR}}$ [19 and 21]. However with the inclusion of finite electron temperature, the change in upper limit to $\theta_{1}$ instead of $\pi / 2$ when $f=f_{\mathrm{LHR}}$ is sufficient to remove the original singularity of $R_{\|, \perp}$ for the cold plasma, as our numerical results show.

The dipole radiation resistance due to the whistler mode is conveniently integrated numerically. The results are given in the next section.

VI. Numerical results and dipole whistler mode radiation efficiency. - To construct numerical plots, (16), (17), (18) and (19) are integrated numerically. In figure 3 , the dipole radiation resistance $R_{\|, \perp}^{t}$ due to the thermal modes is plotted against the normalized frequency $f / f_{\mathrm{He}}$ for the range $0.98>f / f_{\mathrm{He}}>0.2$ and for two different values of $f_{0} / f_{\mathrm{He}}\left(f_{0} / f_{\mathrm{He}}=5,10\right)$. The curves are parametric in the normalized dipole length $h_{0}=2 \pi f_{\mathrm{He}} h / c$; the electron temperature $T_{\mathrm{e}} \simeq 10^{4} \mathrm{~K}$ is used. Figure 4 is a plot for the dipole radiation resistance due to the whistler mode for the same parameters used in figure 3. For the case of $f=f_{\mathrm{LHR}}\left(f_{\mathrm{LHR}} \approx 0.02 f_{\mathrm{He}}\right)$, a few numerical values of 
TABLE I

Radiation resistance at $f=f_{\mathrm{LHR}}, h_{0}=0.05$

$\begin{array}{clcccc}f_{\mathrm{o}} / f_{\mathrm{He}} & f_{\mathrm{LHR}} / f_{\mathrm{He}} & R_{\|}^{\mathrm{w}}(\mathrm{ohms}) & R_{\perp}^{\mathrm{w}}(\mathrm{ohms}) & R_{\|}^{\mathrm{t}}(\mathrm{ohms}) & R_{\perp}^{\mathrm{t}}(\mathrm{ohms}) \\ 10 & 0.0233 & 6.25 \times 10^{4} & 9 \times 10^{4} & 5.8 \times 10^{4} & 8.2 \times 10^{3} \\ 5 & 0,019 & 1.25 \times 10^{4} & 2 \times 10^{4} & 2.1 \times 10^{4} & 7.4 \times 10^{2}\end{array}$

TABLE II

Summary of the whistler mode efficiency of a dipole antenna in the frequency range $f_{\mathrm{He}}>f \gg f_{\mathrm{LHR}}$ and $f=f_{\mathrm{LHR}}$

a) $0.2 f_{\mathrm{He}}<f<0.975 f_{\mathrm{He}}$

\begin{tabular}{|c|c|c|}
\hline$f_{0} / f_{\mathrm{He}}$ & $h_{0}$ & $\eta_{\|}^{\mathrm{w}}$ \\
\hline 5 & 0.05 & $0 \%$ \\
\hline & 0.5 & $89-100 \%$ \\
\hline 10 & $\begin{array}{l}0.05 \\
0.5\end{array}$ & $\begin{array}{c}80-94 \% \\
99 \%\end{array}$ \\
\hline
\end{tabular}

$\frac{\eta_{\perp}^{\mathrm{w}}}{86-88 \%}$
$95 \%$
$95 \%$
$100 \%$

$\frac{\eta_{\|}^{\mathrm{t}}}{20-30} \%$
$0-11 \%$
$6-20 \%$
$1 \%$

$\frac{\eta_{\perp}^{\mathrm{t}}}{12-14} \%$
$5 \%$
$5 \%$
$0 \%$

b) $f \simeq f_{\mathrm{LHR}}, h_{0}=0.05$

$\begin{array}{cc}f_{\mathrm{o}} / f_{\mathrm{He}} & f / f_{\mathrm{He}} \\ 5 & 0.019 \\ 10 & 0.023\end{array}$
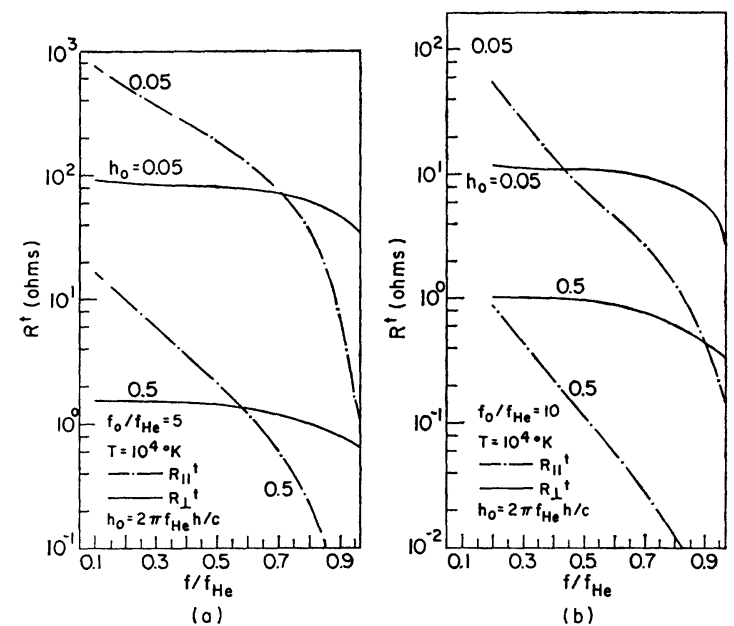

FIG. 3. - VLF dipole radiation resistance of the electron thermal mode as a function of normalized antenna length, antenna orientation, and driving frequency for $f_{q} \ll f<f_{\text {He. }}$. Two normalized densities are considered : a) $f_{0} / f_{\mathrm{He}}=5$ and b) $f_{0} / f_{\mathrm{He}}=10$.

the dipole radiation resistance for $h_{0}=0.05$ and $f_{0} / f_{\mathrm{He}}=5,10$ are summarized in table $\mathrm{I}$. In constructing table I, the value $h / r_{0}=10^{3}$ is used and an electronproton plasma is assumed.

Using the results of figures 3 and 4 and table I along with the definitions: $\eta^{\mathbf{w}}=R^{\mathbf{w}} /\left(R^{\mathbf{w}}+R^{t}\right)$, $\eta^{t}=R^{t} /\left(R^{\mathrm{w}}+R^{t}\right)\left(R^{\mathrm{w}}\right.$ and $R^{\mathrm{t}}$ stand for radiation resistance of the whistler mode and the propagating thermal modes, respectively), the whistler mode radiation efficiency of a dipole antenna has been summarized

$$
\begin{aligned}
& \eta_{\perp}^{\mathrm{w}} \\
& \sim \overline{76} \% \\
& \stackrel{\eta_{\|}^{2}}{\sim 62 \%} \\
& \begin{aligned}
& \frac{\eta_{\perp}^{\mathrm{t}}}{24 \%} \\
\sim & \sim 10 \%
\end{aligned} \\
& \sim 90 \% \\
& \sim 50 \%
\end{aligned}
$$

in table II. The data of table II indicate that as $h_{0}, \varphi_{0}$ (the orientation angle) and $f_{0} / f_{\mathrm{He}}$ increase, the radiation efficiency for the whistler mode increases.

VII. Discussion and concluding remarks. - Using a linear full electromagnetic wave theory, an analysis has been made to calculate the input impedance of an electric antenna embedded in a uniform, lossless, unbounded warm magnetoplasma. The warm magnetoplasma is assumed to consist of warm electrons and cold ions. Specifically, the formal solutions for the input impedance for a "short 》 antenna (in the sense that the triangular current distribution is valid) oriented either parallel or perpendicular to the static magnetic field have been derived. In the VLF range $f_{\mathrm{He}} \geqslant f \geqslant f_{\mathrm{LHR}}$, the dispersion equation for the warm magnetoplasma system is analyzed in detail and the various modes for propagation are specified. Using the results from the analysis of the dispersion equation, the dipole radiation resistances due to the whistler mode and the thermal modes (the TMW mode and the electron thermal mode) have been obtained in integral form. Numerical results for the dipole radiation resistance due to the various modes have been obtained for the case in which the ionic component consists entirely of protons. These results are either plotted (Fig. 3 and 4) or listed in table I. From these numerical results, we are able to estimate the VLF radiation efficiency into the whistler mode for an electric antenna embeded in a warm magnetoplasma. From our numerical data (Table II), it can be concluded that for the VLF 


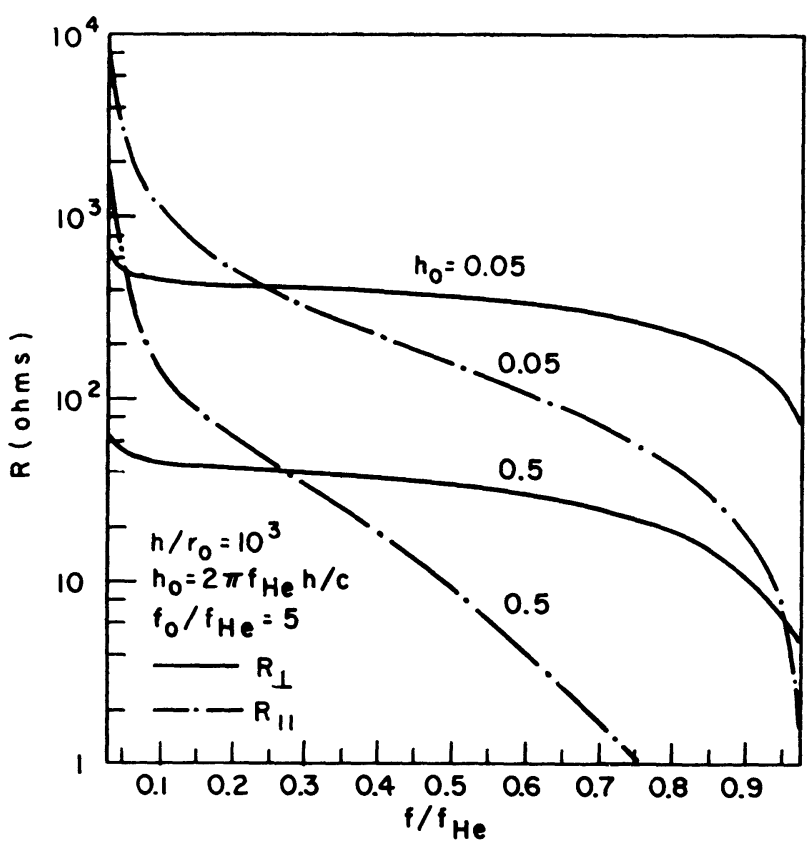

(a)

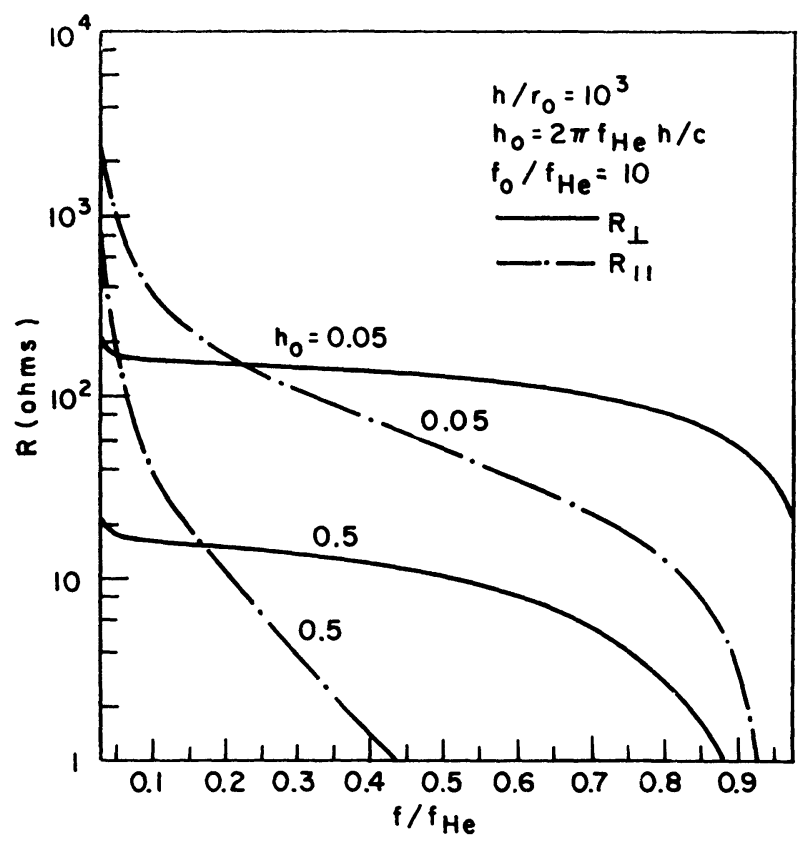

(b)

FIG. 4. - VLF dipole radiation resistance of the whistler mode as a function of normalized antenna length, antenna orientation and driving frequency for $f_{\mathrm{LHR}}<f<f_{\mathrm{He}}$. Two normalized densities are considered : a) $f_{0} / f_{\mathrm{He}}=5$ and b) $f_{0} / f_{\mathrm{He}}=10$.

dipole radiation in the magnetospheric plasma, the whistler mode radiation efficiency $\eta^{\mathrm{w}}$ is usually greater than $70 \%$, although at $f \simeq f_{\text {LHR }} \eta^{\mathrm{w}}$ may be as low as $38 \%$. In general $\eta^{\mathrm{w}}$ increases with increase of anyone of the factors: dipole length, plasma density and $\varphi_{0}$. In most cases $\eta_{\perp}^{\mathrm{w}}$ is greater than $\eta_{\|}^{\mathrm{w}}$ by at least $10 \%$.

In the present investigation, we have not considered any effects from the short range collisions in the plasma. With the inclusion of a finite collision, $\varepsilon_{v}=1-\sum X_{s} /\left(1+v Y_{s}-j Z_{s}\right), \quad$ where $\quad Z_{s}=c_{s} / \omega$ is the normalized collision frequency for $« s »$ species. The relevant generalization for the case $Z_{s} \neq 0$ is not difficult. However in the magnetosphere, the probabilities for occurrence of short-range collisions are very small and it can be shown that these collisions are less important than the thermal effects in determining the amount of VLF dipole power radiated into the whistler mode (see p. 78 of [21]).

In the frequency range $f_{\mathrm{LHR}}>f>f_{\mathrm{Hp}}$ it can be shown that under the condition $\tilde{u}^{2} \ll 1$, the real angle $\theta_{2}$, defined in (12) no longer exists. An analysis of the dispersion eq. (11) yields : 1) for the wave normal angles $0<\theta<\pi / 2$, the whistler mode is practically unaffected by the finite temperature ; 2) for the wave normal angles $\theta_{1}<\theta<\pi / 2$ a propagating electron thermal mode exists in addition to the whistler mode.

The apparent existence of a propagating thermal mode over such a wide frequency range is in conflict with known results, obtained from the Vlasov equation, that perpendicularly propagating thermal modes can exist only at discrete frequencies (generally multiples of the ion gyrofrequencies) in the range $f_{\mathrm{LHR}}>f>f_{\mathrm{Hp}}$ [32]. This conflict in theoretical results can be resolved by including the effects of ion temperature. For example, [34] have considered warm ions as well as electrons and have derived an approximate expression for the refractive index of the low frequency thermal modes propagating across the magnetic field (eq. (5.48) of [34]). Their approximate expression shows that the square of the thermal mode refractive index becomes negative over the frequency range $f_{\mathrm{LHR}}>f>f_{\mathrm{Hp}}$, indicating that a thermal mode cannot propagate. Thus it can be concluded that in the frequency range $f_{\mathrm{LHR}}>f>f_{\mathrm{Hp}}$ the existence of the electron thermal mode in the range $\theta_{1}<\theta<\pi / 2$ is predicted from (11) only as a result of our neglect of the ion temperature in our model.

Since over the frequency range $f_{\mathrm{Hp}}<f<f_{\mathrm{LHR}}$ it can be shown that the whistler refractive index is essentially unaffected by the finite temperature, the VLF dipole radiation resistance will be given to a good approximation by the results obtained for the cold plasma case [19].

It is important to note that in the present investigation, we have assumed the plasma is entirely uniform and have therefore not considered the effects of a finite plasma sheath surrounding the antenna. The antenna-sheath problem is in general a nonlinear one [28] and it is likely that a portion of the dipole power will be lost in nonlinear mechanisms within the sheath. Thus for a more accurate calculation of the dipole whistler mode radiation efficiency, the nonlinear antenna-sheath interaction should be one of the most important problems to investigate.

Acknowledgments. - This research was supported by the National Aeronautics and Space Administration under grant NGL-05-020-008. 


\section{References}

[1] Kogelnik (H.), J. Res. NBS, D (Radio Propagation), 1960, 64, 515 .

[2] KogelniK (H.), Proc. 4th Int. Conf. Ionization Phenomena in Gases, Vol. II, 1960, North-Holland Publ. Co., Amsterdam.

[3] Kogelnik (H.), Motz (H.), Electromagnetic Theory and Antennas, Proc. Symposium at Copenhagen, 1962, 1963, Pergamon Press, Oxford.

[4] Kuehl (H.), Phys. Fluids, 1962, 5, 1095.

[5] Staras (H.), IEEE Trans. Ant. Prop., 1964, AP-12, 695.

[6] Weil (H.), Walsh (D.), IEEE Trans. Ant. Prop., 1964, AP-12, 297.

[7] Weil (H.), Walsh (D.), IEEE Trans. Ant. Prop., 1965, AP-13, 21.

[8] Seshadri (S.), IEEE Trans. Ant. Prop., 1965, AP-13, 819.

[9] Seshadri (S.), Canad. J. Phys., 1968, 46, 283.

[10] Seshadri (S.), Tuan (H.), Proc. IEE, 1965, 112, 2192.

[11] Ament (W.), Katzin (J.), Katzin (M.), Koo (B.), Radio Sci., 1964, 68D, 379.

[12] Wu (C.), IEEE Trans. Ant. Prop., 1963, AP-11, 681.

[13] Balmain (K.), IEEE Trans. Ant. Prop., 1964, AP-12, 605.

[14] Galejs (J.), IEEE Trans. Ant. Prop., 1968, AP-16, 378.

[15] Galejs (J.), IEEE Trans. Ant. Prop., 1968, AP-16, 728.

[16] Duff (G.), MitTra (R.), Radio Sci., 1970, 5, 81.

[17] Wang (T.), Bell (T.), Radio Sci., 1969, 4, 167.

[18] Wang (T.), Bell (T.), IEEE Trans. Ant. Prop., 1969, AP-17, 824.

[19] WANG (T.), Bell (T.), Radio Sci., 1970, 5, 605.

[20] Bell (T.), Wang (T.), IEEE Trans. Ant. Prop., 1971, AP-19, 517.
[21] WANG (T.), Ph. D. Thesis, Stanford University, Stanford, Calif., 1970.

[22] Kieburtz (R.), Radio Sci., 1964, 68D, 795.

[23] Cheng (H.), Cheng (D.), IEEE Trans. Ant. Prop., 1966, AP-14, 71.

[24] Tuan (H.), Seshadri (S.), IEEE Trans. Ant. Prop., 1965, AP-13, 71.

[25] Deschamps (G.), Kesler (O.), Radio Sci., 1967, 2, 757.

[26] Kuehl (H.), Phys. Fluids, 1963, 6, 1465.

[27] Wait (J.), Canad. J. Phys., 1964, 42, 1760.

[28] BACHYNSKi (M.), Sources in unbounded plasmas, RCA Victor Res. Rept. No. 7-801-50, RCA Victor Co., Ltd., Montreal, Canada, 1966.

[29] Kaiser (T.), Tunaley (J.), Space Sci. Rev., 1968, 8, 32.

[30] Chen (F.), Plasma Diagnostic Techniques, 1965, Academic Press, New York.

[31] Mlodnosky (R.), Garriott (O.), Proc. Int. Conf. on the Ionosphere, Inst. Phys. and Phys. Soc., 1962, London.

[32] Stix (T.), The Theory of Plasma Waves, 1962, McGraw-Hill Book Co., Inc., New York.

[33] Ratcliffe (J.), The Magnetoionic Theory and Its Application to the Ionosphere, 1959, Cambridge University Press, London.

[34] Allis (W.), Buchsbaum (S.), Bers (A.), Waves in Anisotropic Plasmas, 1963, MIT Press, Cambridge, Mass.

[35] Burnside (W.), Panton (A.), The Theory of Equations with an Introduction to the Theory of Binary Algebraic Terms, 1960, Dover Publ. Co., Inc., New York. 